\title{
CRECIMIENTO Y VARIABLES ECOFISIOLÓGICAS EN BRINZALES DE Ziziphus thyrsiflora Y Myroxylon balsamum CULTIVADOS EN VIVERO
}

\section{GROWTH AND ECOPHYSIOLOGICAL VARIABLES IN SEEDLINGS OF Ziziphus thyrsiflora AND Myroxylon balsamum NURSERY GROWN}

\author{
Dolores Rosalía Mendoza Chumo, Gibelly Lisbeth Naranjo Moran, Esteban Chirino Miranda,
}

Facultad Ciencias Agropecuarias, Universidad Laica Eloy Alfaro de Manabí, Manabí, Ecuador

Email: doloresmendozam04@gmail.com, gibelynaranjom04@gmail.com, esteban.chirino@uleam.edu.ec

\section{Información del artículo}

Tipo de artículo: Artículo original

Recibido: 11/08/2021

Aceptado:

$15 / 12 / 2021$

Licencia: CC BY-NC-SA 3.0

Revista ESPAMCIENCIA 12(2):95-106

DOI:

https://doi.org/10.5 1260/revista_espam ciencia.v12i 2.268

\section{Resumen}

El objetivo del presente estudio fue evaluar la estrategia en crecimiento, control estomático y potencial hídrico de brinzales de ébano (Ziziphus thyrsiflora) y bálsamo (Myroxylon balsamum) en condiciones de humedad óptima del substrato en vivero. Brinzales de 18 meses fueron trasplantados a macetas de $5 \mathrm{~L}$ y cultivados durante tres meses en condiciones de vivero. Al finalizar este período se evalúo: altura del tallo, diámetro en la base del tallo, tasa de crecimiento relativo $(T C R)$ en altura y diámetro, profundidad máxima de enraizamiento, contenido de agua en el suelo $(S W C)$, potencial hídrico al alba $\left(\Psi_{p d}\right)$, conductancia estomática $(G \mathrm{~s})$, y varios índices del estado hídrico de las hojas (contenido hídrico relativo, $C H R$; contenido de agua para la saturación, $C A S$; déficit de agua para la saturación, $D A S$ ). Los resultados indicaron que $Z$. thyrsiflora mostró mayor altura, diámetro y $T C R$ en diámetro; no observándose diferencias entre especies respecto a la profundidad máxima de enraizamiento $(50 \mathrm{~cm})$. Aunque las dos especies fueron regadas al mismo tiempo con igual dosis, Z. thyrsiflora registró inferior $S W C$ que $M$. balsamum cuando $\Psi_{p d}$ fue medido; lo cual se reflejó en un menor $\Psi_{p d}(Z$. thyrsiflora, $\Psi_{p d}=-0,74 \mathrm{MPa} ; M$. balsamum, $\left.\Psi_{p d}=-0,52 \mathrm{MPa}\right)$ y mayor $C A S$ (Z. thyrsiflora, $C A S=0,27 \mathrm{~g} \mathrm{H}_{2} \mathrm{O} \mathrm{g}^{-1}$ $P S ; M$. balsamum, $\left.C A S=0,14 \mathrm{~g} \mathrm{H}_{2} \mathrm{O} \mathrm{g}^{-1} P S\right)$. $S W C$ correlacionó positivamente con el $\Psi_{p d}$ y negativamente con el CAS. Z. thyrsiflora mantuvo valores superiores de Gs $\left(191,10 \mathrm{molH}_{2} 0 . \mathrm{m}^{-2} . \mathrm{s}^{-1}\right)$; respecto a M. balsamum $(100,84$ $\left.\mathrm{molH}_{2} 0 . \mathrm{m}^{-2} \cdot \mathrm{s}^{-1}\right)$. Z. thyrsiflora mostró una estrategia anisohídrica y M. balsamum una estrategia isohídrica.

Palabras clave: tasa de crecimiento relativo, potencial hídrico, conductancia estomática, isohídrico, anisohídrico.

\footnotetext{
Abstract

The objective of the present study was to evaluate the growth strategy, stomatal control and water potential in seedlings of ebony (Ziziphus thyrsiflora) and balsam (Myroxylon balsamum) in optimum humidity conditions of the substrate in the nursery. 18-month-old seedlings were transplanted into $5 \mathrm{~L}$ pots and grown for three months in nursery conditions. At the end of this period, the following variables were evaluated: stem height, diameter at the base of the stem, relative growth rate $(T C R)$ in height and diameter, maximum rooting depth, soil water content $(S W C)$, predawn water potential $\left(\Psi_{p d}\right)$, stomatal conductance $(G s)$, and various indices of leaf water status (relative water content, $C H R$; water content for saturation, $C A S$; water deficit for saturation, $D A S$ ). The results indicated that $Z$. thyrsiflora showed greater height, diameter and TCR in diameter; not observing differences between species regarding the maximum rooting depth $(50 \mathrm{~cm})$. Although the two species were watered at the same time with the same dose, Z. thyrsiflora registered a lower SWC than M. balsamum when $\Psi_{p d}$ was measured; which was reflected in a lower $\Psi_{p d}\left(Z\right.$. thyrsiflora, $\Psi_{p d}=-0.74 \mathrm{MPa} ; M$. balsamum, $\Psi_{p d}=-0.52$ $\mathrm{MPa}$ ) and higher $C A S$ (Z. thyrsiflora, $C A S=0.27 \mathrm{~g} \mathrm{H}_{2} \mathrm{O} \mathrm{g}^{-1} P S ;$. . balsamum, $C A S=0.14 \mathrm{~g} \mathrm{H}_{2} \mathrm{O} \mathrm{g}^{-1} P S$ ). SWC correlated positively with $\Psi_{p d}$ and negatively with $C A S$. Z. thyrsiflora maintained higher $G s$ values (191.10 $\left.\mathrm{molH}_{2} 0 . \mathrm{m}^{-2} \cdot \mathrm{s}^{-1}\right)$; with respect to M. balsamum $\left(100.84 \mathrm{molH}_{2} 0 . \mathrm{m}^{-2} \cdot \mathrm{s}^{-1}\right)$. Z. thyrsiflora showed an anisohydric strategy and $M$. balsamum an isohydric strategy.
}

Keywords: relative growth rate, water potential, stomatal conductance, isohydric, anisohydric. 


\section{INTRODUCCIÓN}

Los bosques secos en Ecuador se encuentran continuos en el litoral y aislados en los valles secos interandinos (Aguirre et al., 2006a), forman parte de la región Tumbesina y ocupan $62000 \mathrm{~km}^{2}$ compartidos entre Ecuador y Perú (Linares-Palomino et al., 2009), lo que representa aproximadamente el 35\% del Ecuador occidental (Aguirre y Geada-López, 2017). Los bosques secos de la región Tumbesina constituyen "El centro de endemismo Tumbesino" y uno de los puntos calientes de biodiversidad del planeta (Myers et al., 2000; Paladines, 2003). A pesar de su alto endemismo y diversidad florística, los bosques secos tropicales siguen estando poco protegidos (Pennington et al., 2006), y se encuentran amenazados por la expansión de la frontera agrícola, el sobrepastoreo, la extracción de madera y los incendios forestales, factores principales que reducen la superficie de dicho ecosistema (Aguirre et al., 2014).

A pesar de la amenaza existente sobre los bosques secos tropicales, existen áreas donde estos bosques han sido poco intervenidos y aún se puede encontrar una gran diversidad de especies leñosas y principalmente de especies endémicas (Aguirre et al., 2006b). Ébano (Ziziphus thyrsiflora Benth) y bálsamo (Myroxylon balsamum L.) son dos especies maderables endémicas de bosque seco que están amenazadas por la deforestación (Aguirre, 2012; Indacochea et al., 2017), destrucción del hábitat y sobrexplotación para extracción de su madera (Payares-Díaz et al., 2014). La especie Z. thyrsiflora pertenece a la familia Rhamnaceae, y se encuentra distribuida en la región Tumbesina. Es un árbol perennifolio de 10-18 $\mathrm{m}$ de altura, presenta espinas opuestas (pareadas) en las ramitas terminales, sus hojas son simples alternas, se reproduce por semillas y es de crecimiento lento (Aguirre, 2012). M. balsamum es una especie perteneciente a la familia Fabaceae, y en Ecuador se encuentra en las provincias de Manabí y Esmeraldas (Indacochea et al., 2017). Es un árbol perennifolio, de 30$35 \mathrm{~m}$ de altura, crece anualmente un promedio de $2,8 \mathrm{~m}$ en altura y $2.4 \mathrm{~cm}$ en diámetro, presenta hojas compuestas de 5 a 10 foliolos alternos, tiene una raíz pivotante y ramificada y es una especie de fácil adaptación (Morales, 2013).

Los estudios en viveros relacionados con las características morfológicas de especies forestales permiten evaluar la dinámica de desarrollo de las especies en condiciones controladas (Muñoz et al., 2011). Así mismo, la tecnología de producción utilizada durante el cultivo en vivero determinará la calidad de la planta forestal producida y su mejor establecimiento en el campo (Chirino et al., 2009; Prieto et al., 2018). Por otra parte, los estudios sobre ecofisiología vegetal, son una herramienta muy útil para ayudar a explicar los procesos involucrados en la respuesta de las especies a diferentes condiciones abióticas (Vilagrosa et al., 2008); y en este contexto, son evaluadas variables de crecimiento, como altura de la planta y diámetro en la base del tallo (Muñoz et al., 2011); así como variables ecofisiológicas relacionadas con el potencial hídrico (Luna-Flores et al., 2012) y la conductancia estomática de las especies (Chirino et al., 2017). Históricamente, las variables relacionadas con el crecimiento de las plantas se han asociado a evaluaciones de las características morfológicas y de calidad de la planta forestal (Muñoz et al., 2015). De igual forma, la medición del contenido hídrico relativo en hoja y del potencial hídrico permiten conocer la capacidad y tolerancia que tienen las especies a las restricciones hídricas (Borjas et al., 2015; Peña-Rojas et al., 2018a); siendo el potencial hídrico y la conductancia estomática dos variables ecofisiológicas asociadas al estado hídrico de la planta (Luna-Flores et al., 2012) y a las estrategias de las especies ante el estrés hídrico (McDowell, 2011).

Comprender la relación que existe entre la planta y el agua permite analizar el estado hídrico de la misma (Ruscitti, 2015), para lo cual existen varios índices como: el contenido hídrico relativo $(C H R)$, el déficit de agua para la saturación $(D A S)$ y la cantidad de agua para la saturación (CAS) (Bacelar et al., 2006). El CHR es una variable complementaria al potencial hídrico $(\Psi)$ con el fin de determinar el estado hídrico de las plantas (Guajardo, 2010), y está asociado al nivel de estrés de las mismas (Ariza, 2017). El DAS se expresa en porcentaje y se produce cuando la planta libera más agua de la que recibe (Čierniková et al., 2021), es utilizado para detectar la tolerancia de las plantas a la escasez temporal del agua (Rybka et al., 2019) y el CAS es la cantidad de agua que le hace falta a la hoja para la saturación (Abd-El-Rahman et al., 1966).

El agua se considera el principal factor limitante del crecimiento en los ecosistemas terrestres naturales y es uno de los factores más importantes promotores del crecimiento en ecosistemas artificiales como los invernaderos (Landis et al., 1989). Durante el período de cultivo en vivero, mantener el contenido de agua del substrato en un rango óptimo juega un papel muy importante en la calidad de la planta producida, por cuanto determina en cierta medida el desarrollo de las plantas y sus características morfológicas (Chirino et al., 2004). Una célula vegetal requiere un contenido de agua entre 85 a 90\% (González et al., 2018). La reducción o el incremento excesivo de contenido de agua en el suelo afecta la actividad de la célula vegetal y el crecimiento de las plantas (Maiti et al., 2016). Estudios previos consultados (Calle y Proaño, 2003; Barrios-Díaz et al., 2006; Abrisqueta, 2010; Torrente y Rojas, 2011) indican que valores de humedad del suelo superiores a $25 \%$ pueden corresponder a valores de humedad óptima del suelo porque no limitan el desarrollo del cultivo; pero 
evidentemente, el rango de humedad óptima del suelo para el cultivo estará en función de las características de cada especie.

Los estudios sobre ecofisiología en especies endémicas de bosques secos tropicales son escasos. En las bases bibliográficas consultadas no se encontraron estudios relacionados con evaluaciones de potencial hídrico y conductancia estomática en Z thyrsiflora y M. balsamum. En este contexto, resulta necesario e importante la investigación científica enfocada a la ecofisiología vegetal (Melgarejo et al., 2010), y especialmente, en especies pertenecientes a bosques secos tropicales como en este caso. Esta investigación tuvo como objetivo evaluar la estrategia en crecimiento, control estomático y potencial hídrico de brinzales de $Z$. thyrsiflora y $M$. balsamum en condiciones de humedad óptima del substrato en vivero, como paso previo a la evaluación de la respuesta de estas especies en condiciones de estrés hídrico. Mediante este estudio es posible lograr una aproximación al conocimiento de los requerimientos hídricos de una especie y su estrategia en el uso del recurso agua. De igual forma, contribuye a mejorar en vivero las características morfológicas y fisiológicas de los brinzales (Luis-Díaz, 2009); mejorar la calidad de los brinzales para la conservación de las especies ante climas cambiantes (De la Riva et al., 2013); y a comprender la dinámica de las comunidades ante factores ambientales y antrópicos (Yates et al., 2014).

\section{MATERIALES Y MÉTODOS}

\section{Período de cultivo en vivero}

El experimento se realizó en el vivero del Gobierno Autónomo Descentralizado (GAD) de Manabí en la localidad de Lodana (coordenadas $1^{\circ} 10^{\prime} 30.19 " \mathrm{~S}$ y $\left.80^{\circ} 23^{\prime} 10.66^{\prime \prime O}\right)$. Para el desarrollo del experimento, fueron adquiridos 70 brinzales con edad de 18 meses, de las especies Ziziphus thyrsiflora Benth (ébano) y Myroxylon balsamum L. (bálsamo), procedentes del bosque protector Cerro Blanco (Región Costa, Ecuador; coordenadas $2^{\circ} 11^{\prime} 00.52^{\prime \prime S}$ y $\left.80^{\circ} 00^{\prime} 58.58^{\prime \prime} \mathrm{O}\right)$.

Cuarenta y uno brinzales de cada especie ébano ( $Z$. thyrsiflora) y Bálsamo ( $M$. balsamum), cultivados en bolsas de polietileno (diámetro: $12,7 \mathrm{~cm}$ y profundidad: $20,3 \mathrm{~cm}$ ), fueron trasplantados a macetas de $5,5 \mathrm{~L}$ (diámetro: $18 \mathrm{~cm}$, profundidad: $21,5 \mathrm{~cm}$ ). Se utilizó como substrato una mezcla de compost de restos vegetales y suelo forestal, con arena de río en proporción $4: 2 \mathrm{~kg} / \mathrm{kg}$. A dicha mezcla se le aplicó una fertilización adicional de $2 \mathrm{~g}$ de fertilizante de liberación lenta (fertilizante Multicote, 14-7-14, 12 meses de longevidad) por litro de substrato. Durante el periodo de cultivo en vivero (13/11/2019 a 06/02/2020), se aplicó un régimen de riego de $25 \mathrm{~mm}$ dos veces por semana, lo que permitió mantener la humedad del substrato en valores superiores a $25 \%$.

\section{Monitoreo del crecimiento}

Durante la fase de cultivo en vivero se midió la altura del tallo $(H s ; \mathrm{cm})$ y el diámetro en la base del tallo $(D ; \mathrm{mm})$ en 15 individuos (indiv.) seleccionados al azar por cada especie (total 30 indiv.). Las mediciones se realizaron una semana después del trasplante $(21 / 11 / 2019)$, y a los 86 días de cultivo en vivero (06/02/2020). Posteriormente se calculó la tasa de crecimiento relativo en altura $(T C R)$ mediante la siguiente expresión matemática: $T C R=(\ln$ $\left(H s t_{2}\right)$ - $\left.\ln \left(H s t_{1}\right)\right) /\left(t_{2}-t_{1}\right)$; donde $H s t_{2}$ y $H s t_{1}$ corresponden a la altura de los brinzales al final ( $t_{2}$ tiempo final) y principio ( $t_{l}$ tiempo inicial) de la etapa de cultivo en vivero. Para determinar la $T C R$ en diámetro se realizó un procedimiento similar.

\section{Crecimiento potencial de raíces}

Considerando la importancia del sistema radical de las plantas en la captación de agua y nutrientes, se realizó una prueba de crecimiento potencial de raíces $(C P R)$. El objetivo de esta tarea fue evaluar la estrategia de crecimiento y morfología del sistema radical de las especies estudiadas en condiciones de humedad óptima. Para ello, se seleccionaron al azar siete indiv. /especie, cultivados en bolsas de polietileno (diámetro: $12,7 \mathrm{~cm}$ y profundidad: $20,3 \mathrm{~cm}$ ), los cuales fueron trasplantados en tubos de PVC (diámetro $11 \mathrm{~cm}$, profundidad $50 \mathrm{~cm}$ ). Se utilizó como substrato arena de río. Los individuos fueron cultivados durante 45 días en condiciones de humedad óptima, es decir, contenidos de agua en el suelo ( $S W C$ ) superiores a $25 \%$. Al finalizar este período, los tubos fueron cortados longitudinalmente y con mucho cuidado, se fue retirando la arena, con el fin de medir la profundidad máxima de enraizamiento.

\section{Medición del potencial hídrico e índices del estado hídrico de las hojas}

Una vez finalizada la fase de cultivo en macetas, se seleccionaron al azar 7 indiv/especie (14 indiv. total), a los cuales se les aplicó un riego (dosis $25 \mathrm{~mm}$ ) el día previo a la medición, con la finalidad de alcanzar niveles de $S W C$ $>25 \%$. En la madrugada del día siguiente, y utilizando la cámara de Sholander (Soil Moisture 3005, Soil moisture Equipment Corp., Santa Bárbara, CA, USA), se midió potencial hídrico al alba $\left(\Psi_{p d} ;-\mathrm{MPa}\right)$ en una ramita con diámetro aproximado entre $2-3 \mathrm{~mm}$. Las mediciones se realizaron antes del amanecer, entre las 4:00-5:30 h. Simultáneamente, se midió la humedad del suelo ( $S W C$ ) utilizando la sonda Theta-probe (Delta-TDevices Ltd., Cambridge, UK).

Después de tomar la muestra para determinar el potencial hídrico, en los mismos individuos se tomaron al azar tres 
hojas en la especie ébano y tres foliolos en la especie bálsamo, con el objetivo de determinar varios índices del estado hídrico en las hojas. Cada muestra (hoja o foliolo), se envolvió en papel de aluminio y las tres fueron depositadas en una funda de plástico con cierre, debidamente etiquetada. Seguidamente las muestras foliares se colocaron en una nevera portátil, a baja temperatura en su interior, mantenida con dos piezas de gel congelado. Posteriormente en el laboratorio, y siguiendo el método descrito por Bacelar et al. (2006), se calcularon las siguientes índices del estado hídrico en las hojas: contenido hídrico relativo en hoja $(\mathrm{CHR} ; \%) \mathrm{CHR}$ $=((P F-P S) /(P T-P S)) \times 100$, contenido de agua para la saturación $\left(C A S ; \mathrm{g} \mathrm{H}_{2} \mathrm{O} \mathrm{g}^{-1} P S\right) C A S=(P T-P F) / P S$ y déficit de agua para la saturación $(D A S ; \%) D A S=(P T$ $P F) /(P T-P S) * 100$, donde $P F, P T$ y $P S$ representan el peso fresco, peso turgente y peso seco de la hoja (o foliolo) respectivamente.

\section{Medición de intercambio de gases}

El mismo día de las mediciones de potencial hídrico, se midió al mediodía intercambio de gases. El día anterior, fueron seleccionados al azar 20 indiv. /especie cultivados en macetas durante tres meses, a los cuales se les aplicó un riego con una dosis de $25 \mathrm{~mm}$, con la finalidad de alcanzar niveles de $S W C>25 \%$. Al día siguiente, en el horario entre las 11:30 a 13:30 h, después que los individuos estuvieran expuestos al menos dos horas a la luz solar, se midió conductancia estomática $\left(G s ; \mathrm{molH}_{2} \mathrm{O} \cdot \mathrm{m}^{-2} \cdot \mathrm{s}^{-1}\right)$, utilizando el porómetro AP4 (Delta-T Devices Ltd., Cambridge, UK). Las mediciones se realizaron bajo las siguientes condiciones ambientales: temperatura $\left(T^{\mathrm{a}}\right) 33,14 \pm 0.11^{\circ} \mathrm{C}$, humedad relativa $(H R) \approx 70 \%$ y densidad de flujo de fotones fotosintéticos (PPFD) 435,4 $\pm 25 \mu \mathrm{mol} \cdot \mathrm{m}^{-2} \cdot \mathrm{s}^{-1}$. Seguidamente se midió la humedad del suelo $(S W C ; \%)$ en cada individuo utilizando la sonda Theta-probe (Delta-T Devices Ltd., Cambridge, UK).

\section{Análisis estadístico}

La comparación de las medias de las variables altura del tallo $(H s ; \mathrm{cm})$, diámetro en la base del tallo $(D ; \mathrm{mm})$, tasa de crecimiento relativo en altura y diámetro $\left(T C R ; \mathrm{y}^{-1}\right)$, contenido de agua en el suelo (SWC; \%), potencial hídrico de las plantas $\left(\Psi_{p d} ;-\mathrm{MPa}\right)$, contenido hídrico relativo en hoja $(C H R ; \%)$, contenido de agua para la saturación (CAS; $\left.\mathrm{g} \mathrm{H}_{2} \mathrm{O} \mathrm{g}{ }^{-1} P S\right)$, déficit de agua para la saturación $(D A S ; \%)$ y conductancia estomática $\left(G s ; \mathrm{molH}_{2} \mathrm{O} . \mathrm{m}^{-2} . \mathrm{s}^{-1}\right)$ fueron realizadas mediante el análisis $t$ de student. El análisis de la relación entre las variables $S W C$, índices del estado hídrico de las hojas (CHR, CAS y $D A S)$ y potencial hídrico al alba, se realizó mediante correlación de Pearson. Las variables cuya unidad de medida es porcentaje, fueron transformadas utilizando la expresión matemática Arcoseno $(\sqrt{\text { variable en } \% / 100})$.

\section{RESULTADOS Y DISCUSIÓN}

\section{Crecimiento en altura del tallo y diámetro en la base del tallo}

Al inicio del experimento (21/11/2019), los brinzales de la especie $Z$. thyrsiflora $(48,4 \pm 2,4 \mathrm{~cm})$ mostraban valores de altura del tallo superiores $(\mathrm{p}<0.001$; gráfico 1$)$ al de los brinzales de la especie M. balsamum $(26,4 \pm 1,4 \mathrm{~cm})$, mostrando una diferencia de $22,0 \mathrm{~cm}$. Al finalizar el período de cultivo en vivero, 86 días después, los brinzales de Z. thyrsiflora superaban en $35,8 \mathrm{~cm}$ ( $\mathrm{p}<0,001$; Gráfico 1) a los brinzales de la especie $M$. balsamum, siendo los valores de altura del tallo $73,0 \pm 3,3 \mathrm{~cm}$ y $37,2 \pm 1,8 \mathrm{~cm}$ respectivamente.

Respecto al diámetro en la base del tallo, Z. thyrsiflora mostró valores superiores que $M$. balsamum, tanto al inicio ( $\mathrm{p}<0.001$; Gráfico 2) como al final $(\mathrm{p}<0.001$; Gráfico 2) del experimento. En Z. thyrsiflora, el diámetro al inicio del experimento fue $6,3 \pm 0,3 \mathrm{~mm}$, mientras que al final era 9,6 $\pm 0,3 \mathrm{~mm}$. En $M$. balsamum, el diámetro fue $5,0 \pm 0,2 \mathrm{~mm}$ y $6,8 \pm 0,2 \mathrm{~mm}$, al inicio y final del experimento respectivamente.
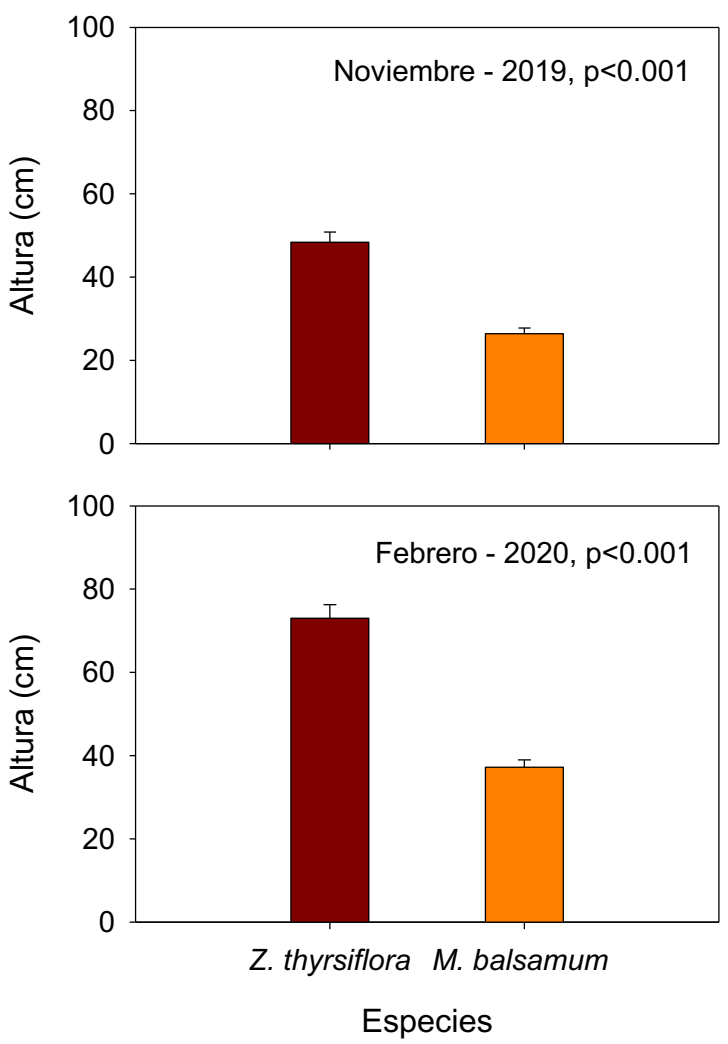

Gráfico 1. Altura del tallo por especies al inicio (Gráfico arriba) y al final del experimento (Gráfico abajo). Resultados análisis $t$ de student. Media \pm error estándar; $\mathrm{N}=15$. 

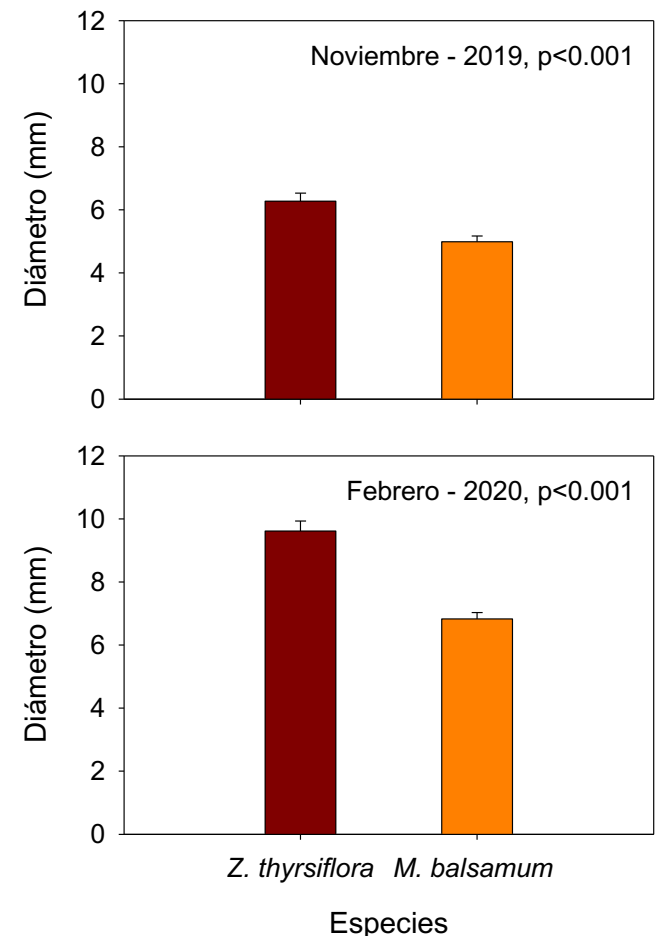

Gráfico 2. Diámetro en la base del tallo por especies al inicio (Gráfico arriba) y al final del experimento (Gráfico abajo). Resultados

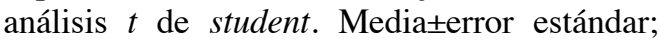
$\mathrm{N}=15$.

En un estudio realizado por Pinargote et al. (2019) en el cual se evaluó el desarrollo inicial de las especies $Z$. thyrsiflora, G. spinosa y $H$. chrysanthus, los resultados indicaron que Z. thyrsiflora mostró mayor crecimiento en altura y diámetro que las otras especies, lo cual es consistente con los resultados del presente estudio. Las especies que presentan un rápido crecimiento se clasifican como especies adquisitivas, mientras que aquellas que crecen lentamente se catalogan como especies conservativas (Zuluaga y Roa, 2017). De acuerdo con Montes-Pulido et al. (2017), las especies adquisitivas de rápido crecimiento presentan mayor cantidad de biomasa como resultado de una mayor captación de luz solar durante la fotosíntesis. Basándose en estas afirmaciones, se podría señalar que $Z$. thyrsiflora es una especie adquisitiva y $M$. balsamun una especie conservativa. No obstante, para una mayor seguridad en la clasificación (especie conservativa o adquisitiva), se debe abarcar el estudio de la planta en su totalidad (De la Riva et al., 2014).

A los tres meses de cultivo en el vivero, no se observaron diferencias entre especies respecto a la tasa de crecimiento relativo (TCR) en altura ( $>>0,05$; gráfico 3$)$, siendo en $Z$. thyrsiflora de $0,005 \pm 0,000 \mathrm{y}^{-1}$ y en $M$. balsamum de $0,004 \pm 0,001 \mathrm{y}^{-1}$. En cambio, la TCR en diámetro de $Z$. thyrsiflora $\left(0,006 \pm 0,000 \mathrm{y}^{-1}\right)$ fue superior $(\mathrm{p}=0,013$; Gráfico 3) a la de M. balsamum $\left(0,004 \pm 0,001 \mathrm{y}^{-1}\right)$. Según
Villar et al. (2008), las especies que tienen mayor TCR son más competitivas en ambientes favorables. En el presente estudio la especie que mostró mayor TCR en diámetro fue Z. thyrsiflora, lo cual significa que incrementó su diámetro en menor tiempo y fue más competitiva que M. balsamum al momento de captar y transformar los recursos.
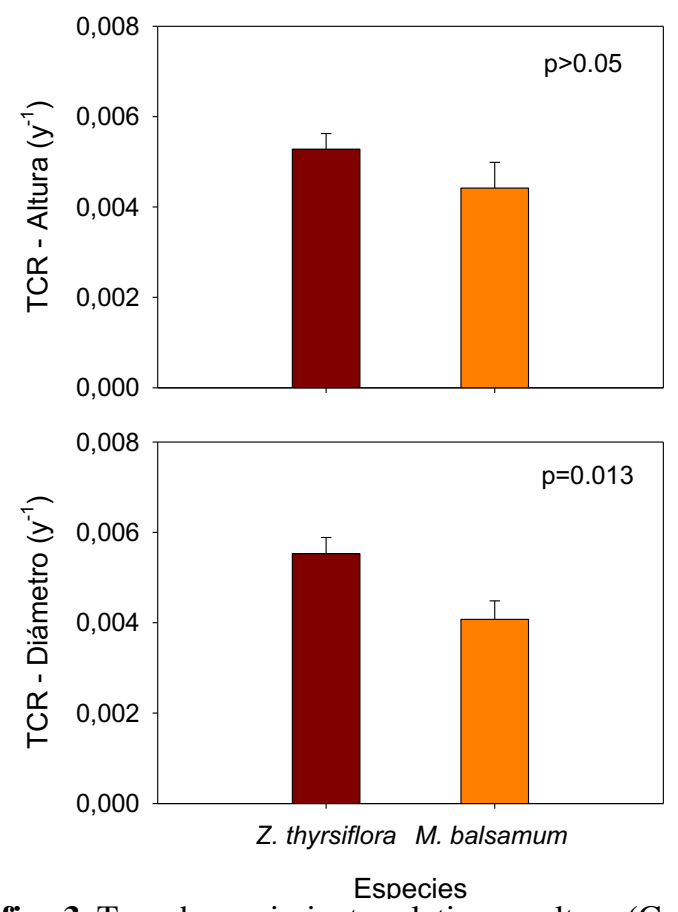

Gráfico 3. Tasa de crecimiento relativo en altura (Gráfico arriba) y en diámetro en la base del tallo (Gráfico abajo). Resultados análisis $t$ de student. Media \pm error estándar; $\mathrm{N}=15$.

\section{Crecimiento potencial de raíces}

Transcurridos 45 días de iniciada la prueba de crecimiento potencial de raíces, no se observaron diferencias en cuanto a la profundidad máxima de enraizamiento. El sistema radical de ambas especies (Z. thyrsiflora y M. balsamum) alcanzó $50 \mathrm{~cm}$ de profundidad, lo cual era la longitud del tubo de PVC. Las especies de bosques secos desarrollan raíces profundas para sobrevivir a severas sequías (Lars et al., 2008). El enraizamiento profundo según Bayar y Deligoz (2016), tiene impactos positivos en las plantas, sobre todo en las especies de bosques secos ya que extraen agua del suelo de capas profundas y con esta estrategia aseguran su supervivencia.

\section{Contenido de agua en el suelo, índices del estado hídrico de las hojas y potencial hídrico de las plantas}

En condiciones no limitantes de humedad en el suelo $(S W C>25 \%)$, previo al momento de realizar las mediciones de potencial hídrico al alba, se observó que $Z$. thyrsiflora presentaba menores valores de $S W C$ respecto a 
M. balsamum ( $\mathrm{p}<0,001$; cuadro 1$)$, a pesar de que en la noche anterior se había realizado un riego con una dosis de $25 \mathrm{~mm}$ a ambas especies. No obstante, a las diferencias observadas en $S W C$, no se encontraron diferencias entre especies respecto al $C H R(\%)$ en hoja ( $>>0,05$; Cuadro 1). Un resultado similar (promedio $C H R=90,2 \%$ ) fue reportado por Peña-Rojas et al. (2018b) en condiciones de alta humedad. Sultana et al. (2019) en experimentos sobre salinidad en dos variedades de cultivos de frijol mungo, indicaron que el $C H R$ en hoja fue superior en los individuos controles que los individuos sometidos a estrés por salinidad y estrés hídrico. El $C H R$ en hoja es utilizado para conocer el estado hídrico de la planta y además indicar el grado de estrés hídrico que soporta en condiciones de sequía (Torres et al., 2019). Por lo anteriormente expuesto, se puede señalar que las especies Z. thyrsiflora y M. balsamum mostraban similar $\mathrm{CHR}$ en hoja en condiciones de $S W C>25 \%$.

Cuadro 1. Contenido de agua en el suelo $(S W C ; \%)$ e índices del estado hídrico de las hojas $(C H R, C A S$ y $D A S)$. Resultados del análisis t de student. Mediaterror estándar; $\mathrm{N}=7$.

\begin{tabular}{|c|c|c|c|c|}
\hline & $\begin{array}{l}S W C \\
(\%)\end{array}$ & $\begin{array}{c}\text { CHR } \\
(\%)\end{array}$ & $\begin{array}{c}C A S \\
\left(\mathrm{~g} \mathrm{H}_{2} \mathrm{O} \mathrm{g}^{-1} P S\right)\end{array}$ & $\begin{array}{l}\text { DAS } \\
(\%)\end{array}$ \\
\hline Z.thyrsiflora & $27,4 \pm 1,6$ & $89,3 \pm 1,5$ & $0,27 \pm 0,04$ & $10,7 \pm 1,5$ \\
\hline M. balsamum & $43,1 \pm 1,3$ & $89,7 \pm 0,8$ & $0,14 \pm 0,01$ & $10,3 \pm 0,8$ \\
\hline Valor $p$ & 0,001 & 0,813 & 0,013 & 0,813 \\
\hline
\end{tabular}

A pesar de no observarse diferencias en el $C H R$ en hoja, en lo que respecta al $C A S\left(\mathrm{~g} \mathrm{H}_{2} \mathrm{O} \mathrm{g}{ }^{-1} P S\right)$, Z. thyrsiflora duplicaba los valores observados en $M$. balsamum ( $\mathrm{p}=$ 0,013 ; Cuadro 1). Este resultado indicó que Z. thyrsiflora requeriría mayor cantidad de agua en la hoja para la saturación, lo cual podría ser una desventaja para soportar ambientes áridos o condiciones de estrés hídrico. Por el contrario, $M$. balsamum necesitaría menor cantidad de agua en sus hojas para la saturación. Borjas et al. (2015), reportaron que la variedad de olivo (Olea europaea L.) Sevillana, al presentar menor $C A S$, tenía mayor capacidad para soportar los ambientes áridos en comparación con la variedad Frantoio, la cual mostraba mayores valores de $C A S$. Especies con menores valores de $C A S$ soportarán mejor los ambientes áridos y con mayor estrés hídrico (Bacelar et al., 2006). Por otra parte, no se observaron diferencias entre especies respecto al DAS (\%) (p>0,05; Cuadro 1). Los resultados relacionados con el $D A S$ demuestran que ambas especies no presentaron mayor pérdida de agua y, por lo tanto, las hojas no se encontraban deshidratadas en el momento de la medición. Rybka et al. (2019) y Mickky et al. (2020) indicaron que el valor del $D A S$ es útil para determinar el grado de deshidratación de los tejidos de las hojas; por consiguiente, cuando las especies se encuentran sometidas a estrés hídrico, el $D A S$ presenta un valor elevado (Tasmina et al., 2016).

En condiciones de $S W C>25 \%$, el potencial hídrico al alba $\left(\Psi_{p d}\right)$ en $Z$. thyrsiflora $(-0,74 \pm 0,04 \mathrm{MPa})$ fue menor ( $\mathrm{p}=0,003$; gráfico 4) al registrado por $M$. balsamum ($0,52 \pm 0,04 \mathrm{MPa}$ ). Ritchie et al. (2010) consideran que el estrés hídrico es muy bajo cuando el valor de potencial hídrico de la planta $(\Psi)$ se encuentra entre 0,0 a $-1,0 \mathrm{MPa}$, y que el estrés hídrico es moderado cuando los valores de $\Psi<-1,0 \mathrm{MPa}$. A pesar de que ambas especies mostraron valores de $\Psi_{p d}$ en el rango de estrés muy bajo $(0,0$ a $-1,0$ $\mathrm{MPa}), Z$. thyrsiflora presentó mayor nivel estrés hídrico con respecto a $M$. balsamum ( $\mathrm{p}=0,003$, gráfico 4).
González et al. (2011) y Peña-Rojas et al. (2018b) señalaron que el potencial hídrico es mayor cuando el $S W C$ presenta valores elevados. El resultado observado en la medición de $\Psi_{p d}$ está relacionado con menores valores de $S W C(\mathrm{p}<0.001$; Cuadro 1) en el momento de las mediciones en $Z$. thyrsiflora $(27,4 \pm 1,6 \%)$ respecto a $M$. balsamum $(43,1 \pm 1,3 \%)$, a pesar de haberse realizado un riego homogéneo la noche previa a la medición. Esta diferencia en $S W C$ puede ser debido a que el riego realizado la noche previa a la medición de $\Psi_{p d}$ no suplió la diferencia existente en la humedad del substrato entre ambas especies. Durante el cultivo de ambas especies en el vivero, se observó que $Z$. thyrsiflora realizaba un consumo de agua superior al de $M$. balsamum, lo cual se evidenciaba en un secado más rápido del substrato en la maceta. Los resultados obtenidos ratificaron la clasificación de $Z$. thyrsiflora como una especie competitiva (Villar et al., 2008), por su alta capacidad de captación del recurso agua. La disminución de la disponibilidad hídrica del sustrato tiene un efecto adverso en el estado hídrico de los individuos (Guajardo, 2010).

El rango de $S W C$ osciló entre $27,4 \%$ en $Z$. thyrsiflora $y$ $43,1 \%$ en $M$. balsamum, lo cual pudiera considerarse un valor ligeramente elevado en la última especie. En la sonda theta-probe, las ecuaciones indicadas por el fabricante para determinar el $S W C$ se utilizan para suelos orgánicos o suelos inorgánicos. En este experimento se utilizó una mezcla de substrato orgánico (compost de restos vegetales y suelo forestal) con substrato inorgánico (arena de río) en proporción 4:2 kg/kg. Esto pudiera ser la causa de los valores relativamente altos de humedad del substrato. López-Seijas et al. (2018) señalaron que las valores de $S W C$ medidos mediante la sonda theta-probe dependen de la textura del suelo. Por otra parte, también se han reportado valores de humedad del substrato registrado con la sonda theta-probe entre 29 y $32 \%$ antes del riego (Sogamoso, 2020). 
Especies

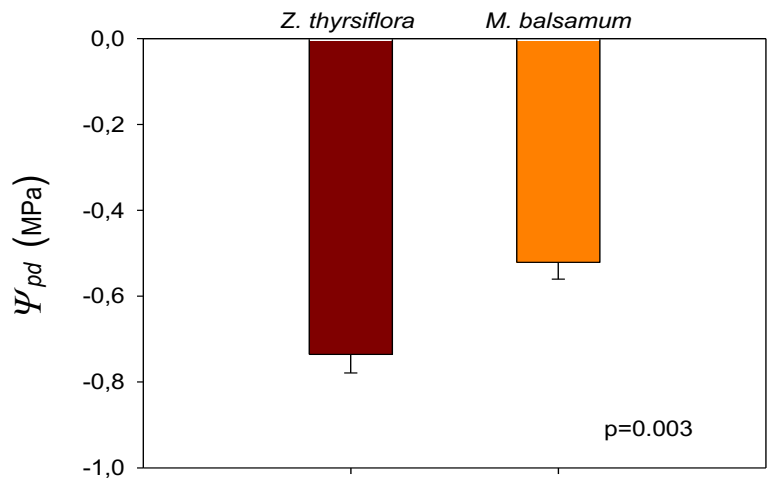

Gráfico 4. Potencial hídrico al alba $\left(\Psi_{p d}\right)$. Resultados del análisis $t$ de student. Mediaterror estándar; $\mathrm{N}=7$.

Relación entre contenido de agua en el suelo, índices del estado hídrico de las hojas y potencial hídrico de las plantas

En condiciones de $S W C>25 \%$ y considerando los valores registrados en diferentes variables en ambas especies $(Z$. thyrsiflora y $\quad M$. balsamum), SWC correlacionó positivamente con el $\Psi_{p d}$ y negativamente con el $C A S$ (Cuadro 2). Por otra parte, el $\Psi_{p d}$ se correlacionó negativamente con el $C A S$; y el $C H R$ en hoja se correlacionó negativamente con el CAS y DAS. Finalmente, el $C A S$ se correlacionó positivamente con el $D A S$. Quintal et al. (2012) indicaron que existe una relación directa entre el $S W C$ y el potencial hídrico de las plantas, observándose altos valores de $\Psi$ en presencia de altos valores de SWC. Sá et al. 2017 y Sultana et al. 2019 indicaron que entre el $D A S$ y el $C H R$ en hoja existe una tendencia inversa; la correlación negativa entre estas variables se debe a que son complementarias, es decir ambas suman el $100 \%$. En el presente estudio no se observó una correlación significativa entre el $S W C$ y las variables $D A S$ y $C H R$, y tampoco entre el $\Psi_{p d}$ y el $D A S$, lo cual puede deberse a que las especies se encontraban en condiciones de humedad del substrato superiores a $25 \%$. Debe tenerse en cuenta que el $D A S$ aumenta cuando las especies presentan déficit hídrico (Gietler et al., 2016), y esto ocurre cuando los valores de $S W C$ son bajos.

Cuadro 2. Correlación de Pearson entre las variables $S W C$, potencial hídrico, índices del estado hídrico de la hoja en las especies Z. thyrsiflora y M. balsamum.

\begin{tabular}{ccccc}
\hline & $\Psi_{p d}$ & $C H R$ & $C A S$ & $D A S$ \\
\hline$S W C$ & $\mathbf{0 , 7 9 9 * *}^{* *}$ & 0.248 & $\mathbf{- 0 , 7 5 5}^{* *}$ & -0.248 \\
$\Psi_{p d}$ & - & 0.032 & $\mathbf{- 0 , 5 9 0}^{*}$ & -0.032 \\
$C H R$ & & - & $\mathbf{0 , 6 9 9}^{* *}$ & $\mathbf{- 1 , 0 0 0}^{* *}$ \\
$C A S$ & & & - & $\mathbf{0 , 6 9 9}$ \\
\hline
\end{tabular}

\section{Conductancia estomática}

La conductancia estomática fue medida en condiciones de altos valores de $S W C, 40 \pm 1,49 \%$ en Z. thyrsiflora y $43 \pm 1,04 \%$ en $M$. balsamum, no observándose diferencias entre las especies $(\mathrm{p}=0,072)$ en $S W C$. Bajo estas condiciones, Z. thyrsiflora $\left(G s=191,1 \pm 23,5 \mathrm{molH}_{2} 0 . \mathrm{m}^{-2} \cdot \mathrm{s}\right.$ $\left.{ }^{1}\right)$ presentó valores de $G s$ superiores ( $\mathrm{p}=0,002$; Gráfico 5) a los registrados por $M$. balsamum (Gs $=100,8 \pm 12,9$ $\left.\mathrm{molH}_{2} 0 . \mathrm{m}^{-2} \cdot \mathrm{s}^{-1}\right)$. Un estudio realizado por Peña-Rojas et al. (2018a) evaluando la respuesta morfo-fisiológicas de plantas de Lithraea caustica (Anacardiaceae) sometidas a restricción hídrica controlada, indicaron que cuando el contenido hídrico del substrato se mantuvo sobre el $40 \%$, no se observaron diferencias en cuanto a intercambio gaseoso, y solo se encontraron diferencias cuando el $S W C$ fue inferior al $30 \%$.

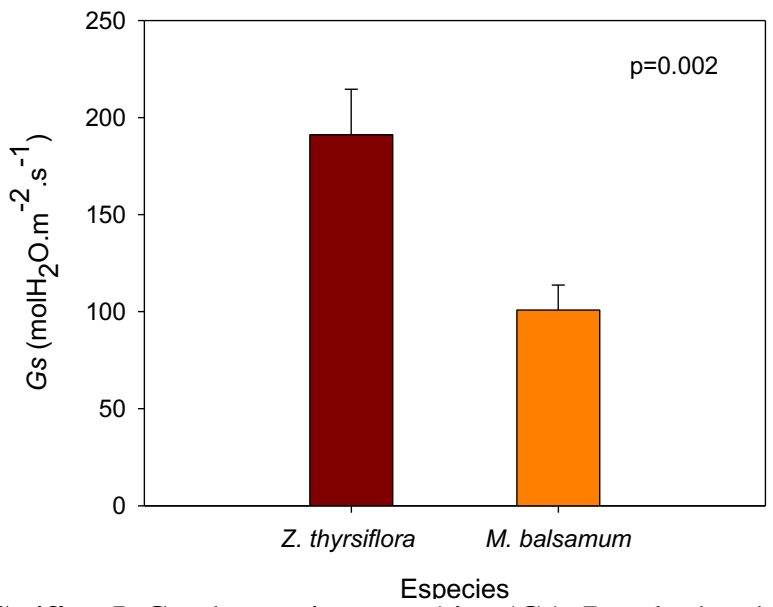

Gráfico 5. Conductancia estomática $(G s)$. Resultados del análisis $\mathrm{t}$ de student. Media土error estándar; $\mathrm{N}=20$.

Las relaciones entre la conductancia estomática, potencial hídrico al alba y la resistencia hidráulica de las hojas están relacionados con el papel de las estomas en la regulación del estado hídrico de la planta (González et al. 2011). Las plantas isohídricas presentan un fuerte control estomático, reduciendo su transpiración y asimilación del carbono; mientras que las plantas anisohídricas no cierran sus estomas y mantienen altos valores de transpiración sin disminuir su capacidad fotosintética (Hugalde y Vila, 2014; Schmidt, 2017). El comportamiento anisohídrico ha sido asociado a una mejor tolerancia al déficit hídrico prolongado (McDowell, et al., 2008). En el presente estudio, la especie Z . thyrsiflora mostro mayores valores de conductancia estomática y menores valores de potencial hídrico, lo que indicó que esta especie adoptó una estrategia anisohídrica. En contraste, M. balsamum desarrolló una estrategia isohídrica, aun en condiciones de humedad óptima, mostrando un bajo consumo de agua mediante la regulación estomática. 
De acuerdo con García y Moreno (2015), la reducción del contenido de agua en el suelo disminuye el potencial hídrico de la hoja y ocasiona el cierre de estomas afectando al intercambio de gases. La relación que existe entre la conductancia estomática y el potencial hídrico de la hoja se debe al estado hídrico de la hoja, por eso se debe tener en cuenta los valores de $S W C$ (Gutiérrez-Gamboa et al., 2019). Los resultados de la presente investigación difieren de los resultados reportados en los estudios previos. La especie M. balsamum, en condiciones de $S W C$ $=43 \%$ reguló la apertura de los estomas manteniendo bajos valores de $G s$; en contraste, Z. thyrsiflora en condiciones de $S W C=40 \%$ mantuvo valores superiores de Gs. Estas diferencias en las estrategias de las especies en cuanto a la regulación estomática deben tener un efecto sobre la captación de recursos, la asimilación de carbono y por consiguiente sobre el crecimiento.

\section{CONCLUSIONES}

En condiciones de humedad óptima en vivero, las especies Ziziphus thyrsiflora y Myroxylon balsamum adoptaron estrategias diferentes respecto al crecimiento y uso del agua disponible en el suelo. En cuanto a crecimiento, $Z$. thyrsiflora demostró ser una especie adquisitiva mostrando un mayor crecimiento tanto en altura como en diámetro en la base del tallo en comparación con $M$. balsamum, lo cual se evaluó como una especie conservativa. En condiciones de $S W C>25 \%$ Z thyrsiflora mostró un mayor consumo de agua del suelo con respecto a M. balsamum, lo que se evidenció en mayores valores de conductancia estomática. Z. thyrsiflora asumió un comportamiento anisohídrico, manteniendo altos valores de conductancia estomática a pesar de mostrar mayores valores de contenido de agua para la saturación de la hoja y menores valores de potencial hídrico, lo que refleja un cierto nivel de estrés hídrico. En cambio, M. balsamun presentó un comportamiento isohídrico, regulando la apertura de sus estomas para conservar la humedad de sus tejidos aún en condiciones de humedad óptima.

\section{AGRADECIMIENTOS}

Agradecemos la colaboración del Gobierno Autónomo Descentralizado (GAD) de la Provincia de Manabí, de la Jefatura de Cooperación de Recursos Forestales y Ambientales (CORFAM), de la Dirección de Gestión Ambiental y del vivero del GAD en Lodana. Esta investigación fue parcialmente financiada por el proyecto "Manejo integral de un paisaje antropogénico para la conservación de biodiversidad, el bioconocimiento y la restauración forestal en Manabî́, el cual es realizado por el Grupo de Ecología Funcional de la Facultad Ciencias Agropecuarias de la Universidad Laica Eloy Alfaro de Manabí (ULEAM).

\section{LITERATURA CITADA}

Abd-El-Rahman, A., Shalaby, A., y Balegh, M. 1966. Water economy of olive under desert conditions. Flora 156: 202-219.

Abrisqueta, V. I. 2010. Balance hídrico y respuesta del melocotonero extratemprano al riego deficitario. Tesis Doctoral. Universidad de Murcia. Murcia, España. Recuperado https://digitum.um.es/digitum/handle/10201/19787

Aguirre, M., Kvist, L., y Sánchez, O. 2006a. Bosques secos en Ecuador y su diversidad. Botánica Económica de los Andes Centrales, 162-187.

Aguirre, M., Linares-Palomino, R., Kvist, L. $2006 \mathrm{~b}$. Especies leñosas y formaciones vegetales en los bosques estacionalmente secos de Ecuador y Perú. Arnaldoa, 13(2): 324-350.

Aguirre, M. 2012. Especies forestales de los bosques secos del Ecuador. Guía dendrológica para su identificación y caracterización. Proyecto Manejo Forestal Sostenible ante el Cambio Climático. MAE/FAO. Quito, Ecuador.

Aguirre, M. Z., Buri, S. D., Geada, L. G., y Betancourt, F. Y. 2014. Composición florística, estructura y endemismo en una parcela permanente de bosque seco en Zapotillo, provincia de Loja, Ecuador. Arnaldoa, 21(1): 165-178.

Aguirre, M., y Geada-López, G. 2017. Estado de conservación de los bosques secos de la provincia de Loja, Ecuador. Arnaldoa, 24(1): 207- 22.

Ariza, A. W. 2017. Respuestas fisiológicas, bioquímicas y rendimiento en tres variedades de papa criolla (Solanum tuberosum grupo Phureja) en déficit hídrico. Tesis de Maestría. Universidad Nacional de Colombia, Facultad de Agronomía. Bogotá, Colombia. Recuperado https://repositorio.unal.edu.co/handle/unal/62084

Bacelar, E., Santos, D., Moutinho-Pereira, J., Gonçalves, B., Ferreira, H., y Correia, C. 2006. Immediate responses and adaptative strategies of three olive cultivars under contrasting water availability regimes: Changes on structure and chemical composition of foliage and oxidative damage. Plant Science, 170: 596605 .

Barrios-Díaz, J., Larios-García, M., Castellanos, J., Alcántar-González, G., Tijerina-Chávez, L., y Rodríguez-Mendoza, M. 2006. Efecto del sistema de 
riego y tensión de humedad del suelo en rendimiento y calidad del ajo. Terra Latinoamericana, 24(1): 75-81.

Borjas, R., Rebaza, D., y Julca, A. 2015. Contenido hídrico de dos variedades de olivo (Olea europaea L.) en el Valle de Cañete, Lima-Perú. Scientia Agropecuaria, 6(3): 147-154.

Bayar, E., y Deligöz, A. 2016. Los efectos de la densidad del semillero sobre la morfología y el potencial de crecimiento de las raíces de las plántulas de espino (Crataegus monogyna Jacq.). Revista turca de silvicultura, 17(1): 7-11.

Calle, K., y Proaño, J. 2003. Determinación de la curva de retención de humedad para los principales tipos de suelo de la Península de Santa Elena, Provincia del Guayas (Presentación de paper). X Congreso Ecuatoriano de la Ciencia del Suelo. Guayaquil, Ecuador. Recuperado de http: //www.secsuelo.org/wpcontent/uploads/2015/06/7.-Determinacion-Curva.p

Chirino, E., Vilagrosa, C, y Rubio, A. 2004. Efectos de la reducción del riego y la fertilización en las características morfológicas de Quercus suber. Cuadernos de la Sociedad Española de Ciencias Forestales, 17: 51-56.

Chirino, E., Vilagrosa, A., Cortina, J., Valdecantos, A., Fuentes, D., Trubat, R., Luis, V.C., Puértolas, J., Bautista, S., Baeza, J., Peñuelas, J., y Vallejo, V. 2009. Ecological restoration in degraded drylands: The need to improve the seedling quality and site conditions in the field. In: Forest Management (Steven P. Grossberg, Ed.) pp 85-158. Nova Science Publishers. New York, USA. ISBN: 978-1-60692-504-1.

Chirino, E., Ruiz-Yanetti, S., Vilagrosa, A., Mera, X., Espinoza, M., y Lozano, P. 2017. Morpho-functional traits and plant response to drought conditions in seedlings of six native species of Ecuadorian Ecosystems. Flora, 233: 58-67.

Čierniková, M., Vykouková, I., Orfánus, T., y Masarovičová, E. 2021. Functional Traits of Plant Species Suitable for Revegetation of Landfill Waste from Nickel Smelter. Applied Sciences, 11(2): 658.

De la Riva, E. G., Tosto, A., Olmo, M., Pérez-Ramos, I. M., Marañón, T., y Villar, R. 2013. Respuestas funcionales comunidades leñosas mediterráneas bajo diferentes escenarios hídrico. 6to Congreso Forestal Español. España.

De la Riva, E., Pérez-Ramos, L., Navarro-Fernández, C., Olmo, M., Marañón, T., y Villar, R. 2014. Rasgos funcionales en el género Quercus: estrategias adquisitivas frente a conservativas en el uso de recursos. Ecosistemas, 23(2): 82-89.

García, L., y Moreno, F. 2015. Respuestas fisiológicas de Theobroma cacao L. en etapa de vivero a la disponibilidad de agua en el suelo. Acta Agron, 65(1): 44-50.

Gietler, M., Nykiel, M., y Zagdańska, B. M. 2016. Changes in the reduction state of ascorbate and glutathione, protein oxidation and hydrolysis leading to the development of dehydration intolerance in Triticum aestivum L. seedlings. Plant growth regulation, 79(3): 287-297.

González, R. H., Cantú, S. I., Ramírez, L., Gómez, M., Pando, M., y López, H. 2011. Potencial hídrico xilemático en cuatro especies arbustivas nativas del noreste de México. Revista Chapingo Serie Ciencias Forestales y del Ambiente, 17: 97-109.

González, R. H., Dueñas, T. H., Cantú, S. I., Cotera, C. M., Ramírez, L. R., y López, H. J. 2018. Relaciones hídricas en arbustos nativos del noreste de México. Revista mexicana de ciencias forestales, 9(50): 121139.

Guajardo, V. A. 2010. Respuestas ecofisiológicas de plantas de Lithraea caustica (Mol.) Hook et Arn. sometidas a restricción hídrica controlada. Tesis de Pregrado. Universidad de Chile, Facultad de Ciencias Forestales y Conservación de la Naturaleza. Santiago de Chile, Chile. Recuperado de http://repositorio.uchile.cl/handle/2250/151596

Gutiérrez-Gamboa, G., Pérez-Donoso, A., Pou-Mir, A., Acevedo-Opazo, C. y Valdés-Gómez, H. 2019. Hydric behaviour and gas exchange in different grapevine varieties (Vitis vinifera L.) from the Maule Valley (Chile). Revista Sudafricana de Enología y Viticultura, 40(2): 3224.

Hugalde, I., y Vila, H. 2014. Comportamiento isohídrico o anisohídrico en vides.... ¿Una controversia sin fin? Revista de Investigaciones Agropecuarias, 40(1): 7582.

Indacochea, B., Parrales, J., Hernández, A., Castro, C., Vera, M., Zhindon, A., y Gabriel, J. 2017. Evaluación de medios de cultivo in vitro para especies forestales nativas en peligro de extinción en Ecuador. AgronCostarricense, 42(1): 63-89.

Landis, T. D. 1989. Irrigation and water management. In: Landis T. D.; Tinus, R.W.; S. E.; McDonald, S.E.; y Barnett, J. P. The Container Tree Nursey Manual, 
Volume 4. Agric. Handbk.674. Washington, DC: U.S, Department of Agriculture, Forest Service: 69-118.

Lars, M., Poorter, L., y Yanguas-Fernández, E. 2008. La disponibilidad hídrica estacional y topográfica en un bosque seco y húmedo tropical y la variación en la morfología de las plántulas arbóreas. Rev. Bol. Ecol. y Cons. Amb. 24: 27-42.

López-Seijas, T., Ustariz, A., Cisnero-Zayas, E., Rodríguez-González, A., Herrera-Puebla, J., y González-Robaina, F. 2018. Calibration of electromagnetic probes for irrigation studies in different agricultural zones. Revista Ingeniería Agrícola, 8(3): 31-39.

Linares-Palomino, R., Kvist, L., Aguirre-Mendoza, Z., y Gonzales-Inca, C. 2009. Diversity and endemism of woody plant species in the Equatorial Pacific seasonally dry forests. Biodiversity and Conservation, 19: 169-185.

Luis-Díaz, V. C. 2009. Calidad de planta de pino canario para reforestación. Métodos de cultivo y respuestas fisiológicas a factores de estrés. Universidad de La Laguna, Servicio de Publicaciones.

Luna-Flores, W., Estrada-Medina, H., Jiménez-Osornio, J., y Pinzón-López, L. 2012. Efecto del estrés hídrico sobre el crecimiento y eficiencia del uso del agua en plántulas de tres especies arbóreas caducifolias. Terra Latinoam, 30(4): 343-353.

Maiti, R., Rodríguez, H. G., y Ivanova, N. S. 2016. Autoecology and ecophysiology of woody shrubs and trees: concepts and applications. John Wiley \& Sons.

McDowell, N. G. 2011. Mechanisms linking drought, hydraulics, carbon metabolism, and vegetation mortality. Plant physiology, 155(3): 1051-1059.

McDowell, N., Pockman, W., Allen, C, Breshears, D. Cobb, N., Kolb, T., Plaut, J., Sperry, J., West, A., Williams, D., y Yepez, E. 2008. Mechanisms of plant survival and mortality during drought: why do some plants survive while others succumb to drought? New Phytol. 178: 719-739.

Melgarejo, L., Romero, M., Hernández, S., Barrera, J., Solarte, M., Suárez, D., Pérez, L., Rojas, A., Cruz, M., Moreno, L., Crespo, S., y Pérez, W. 2010. Experimentos en fisiología vegetal. Departamento de Biología.

Mickky, B., Aldesuquy, H. y Elnajar, M. 2020. Effect of drought on yield of ten wheat cultivars linked with their flag leaf water status, fatty acid profile and shoot vigor at heading. Physiol Mol Biol Plants 26: 11111117.

Montes-Pulido, C., Parrado-Rosselli, A., y ÁlvarezDávila, E. 2017. Tipos funcionales de plantas como estimadores de carbono en bosque seco del Caribe colombiano. Revista Mexicana de Biodiversidad, 88: 241-249.

Morales, R. 2013. El estoraque (Myroxylon balsamum (L.) Harms). Tesis de Pregrado. Universidad Nacional de Cajamarca, Facultad de Ciencias Agrarias. Jaén, Perú. Recuperado http://repositorio.unc.edu.pe/handle/UNC/419

Muñoz, H., García, J., Coria, V., Orozco, G., y Muñoz, Y. 2011. Características morfológicas de plántulas de dos especies forestales tropicales propagadas en contenedores biodegradables y charolas styroblock. Revista mexicana de ciencias forestales, 2(8): 21-34.

Muñoz, H., Sáenz, J., Coria, V., García, J., Hernández, J., y Manzanilla, G. 2015. Calidad de planta en el vivero forestal La Dieta, Municipio Zitácuro, Michoacán. Revista mexicana de ciencias forestales, 6(27):72-89.

Myers, N., Mittermeier, R., Mittermeier, C., Da Fonseca G., y Kent, J. 2000. Biodiversity hotspots for conservation priorities. Nature 403: 853-858.

Paladines, R. 2003. Propuesta de conservación del Bosque seco en el Sur de Ecuador. Lyonia, 4(2): 183-186.

Payares-Díaz, I., Mario-Contreras, O. A., Medrano-Vélez, M. A., y Millán-Romero, E. 2014. Germinación y desarrollo de plántulas de Myroxylon balsamum (L.) Harms en el Departamento de Sucre. Colombia Forestal, 17(2): 193-201.

Pennington, R., Lewis, G., y Ratter, J. 2006. Neotropical Savannas and Seasonally Dry Forests Plant Diversity, Biogeography, and Conservation. Boca Ratón, FL: Taylor and Francis.

Peña-Rojas, K., Donoso, S., Pacheco, C., Riquelme, A., Rodrigo, G., Guajardo, A., y Duran, S. 2018a. Respuestas morfo-fisiológicas de plantas de Lithraea caustica (Anacardiaceae) sometidas a restricción hídrica controlada. Bosque, 39(1): 27-36.

Peña-Rojas, K., Donoso, S., Gangas, R., Durán, S., y Ilabaca, D. 2018b. Efectos de la sequía en las relaciones hídricas, crecimiento y distribución de biomasa en plantas de Peumus boldus Molina (Monimiaceae) cultivadas en vivero. Interciencia, 43(1): 36-42. 
Pinargote, C., Quiñonez, H., Mero, J., Ramos, R., y Cabrera, V. 2019. Desarrollo inicial en plantaciones de Ziziphus thyrsiflora, Geoffroea spinosa Jacq $y$ Handroanthus chrysanthus Jacq. En Jipijapa, Manabí, Ecuador. UNESUM-Ciencias. Publicación cuatrimestral, 3: 73-84.

Prieto, J., Duarte, A., Goche, J., González, M., y Pulgarín, M. 2018. Supervivencia y crecimiento de dos especies forestales, con base en la morfología inicial al plantarse. Revista mexicana de ciencias forestales, 9(47): 151-168.

Quintal, O. W., Pérez-Gutiérrez, A., Latournerie, M. L., May-Lara, C., Ruiz, S. E., y Martínez, C. A. 2012. Uso de agua, potencial hídrico y rendimiento de chile habanero (Capsicum chinense Jacq.). Revista fitotecnia mexicana, 35(2): 155-160.

Ritchie, G. A., Landis, T. D., Dumroese, R. K., y Haase, D. L. 2010. Handling and Shipping. Seedling Processing, Storage and Outplantig. Chapter 2 Assessing Plant Quality. The container tree nursery manual, 19-82.

Rybka, K., Janaszek-Mańkowska, M., Siedlarz, P., y Mańkowski, D. 2019. Machine learning in determination of water saturation deficit in wheat leaves on basis of $\mathrm{Chl}$ a fluorescence parameter. Photosynthetica, 57(1): 226-230.

Ruscitti, M. 2015. Capítulo 2. El agua en la planta. Cultivo en hidroponía, 34.

Sá, F. D., Gheyi, H. R., Lima, G. D., Paiva, E. D., Fernandes, P. D., Moreira, R. C., Silva, L., y Ferreira, N. 2017. Water relations and gas exchanges of West Indian cherry under salt stress and nitrogen and phosphorus doses. Journal of Agricultural Science, 9(10): 168-177.

Schmidt, C. P. 2017. Efecto de distintos niveles de riego sobre la conductividad hidráulica en vides isohídricas y anisohídricas. Trabajo de Pregrado. Escuela de Pregrado, Universidad de Chile, Facultad de Ciencias Agronómicas. Santiago de Chile, Chile. Recuperado de: https://repositorio.uchile.cl/handle/2250/150999

Sogamoso, H. J. 2020. Estudio preliminar de la respuesta fisiológica del lulo sin espinas (Solanum quitoense var. quitoense) expuesto a diferentes niveles de radiación durante la etapa vegetativa en la Sabana de Bogotá. Trabajo de Pregrado. Universidad Militar Nueva Granada, Facultad de Ciencias Básicas y Aplicadas Tecnología en Horticultura. Bogotá, Colombia. Recuperado de: http://hdl.handle.net/10654/35950.
Sultana, M. S., Halim, M., Hossain, F., Karim, M. A., y Hossain, M. T. 2019. Effects of Sodium Chloride Salinity on Water Relations and Ion Accumulation in Two Mungbean Varieties Differing in Salinity Tolerance. Journal of the Asiatic Society of Bangladesh, Science, 45(1): 45-54.

Tasmina, T., Khan, A. R., Karim, A., Akter, N., y Islam, R. 2016. Physiological changes of wheat varieties under water deficit condition. Bangladesh Agronomy Journal, 19(2): 105-114.

Torrente, A. y Rojas, Y. 2011. Determinación del punto óptimo de riego en palma de aceite en la hacienda Ariguanu, Departamento del Cesar. Revista Ingeniería y Región, 8: 1-6.

Torres, I., Sánchez, M. T., Benlloch-González, M., y Pérez-Marín, D. 2019. Soporte de decisiones de riego basado en la determinación del contenido de agua relativa de la hoja en olivar mediante espectroscopia de infrarrojo cercano. Ingeniería de biosistemas, 180: $50-58$

Vilagrosa, A., Chirino, E., Vallejo, V. R., Cortina, J., Rubio, E., Trubat, R., y Gil-Pelegrin, E. 2008. El papel de la ecofisiología en la restauración forestal de ecosistemas mediterráneos. Forest Systems, 14(3): 446-461.

Villar, R., Ruiz-Robleto, J., Quero, J. L., Poorter, H.; Valladares, F. y Marañón, T. 2008. Tasas de crecimiento en especies leñosas: aspectos funcionales e implicaciones ecológicas. Ecología del bosque mediterráneo en un mundo cambiante, 191-227.

Yates, M. L., Andrew, N. R., Binns, M., y Gibb, H. 2014. Morphological traits: predictable responses to macrohabitats across a $300 \mathrm{~km}$ scale. PeerJ, 2: 271.

Zuluaga, N., y Roa, D. C. 2017. Curvas de crecimiento y análisis de rasgos funcionales de especies arbóreas y arbustivas del área de propagación vivero "La Florida" Jardín Botánico de Bogotá José Celestino Mutis. Tesis de Pregrado. Universidad Distrital Francisco José de Caldas, Facultad del Medio Ambiente y Recursos Naturales. Bogotá, Colombia. Recuperado de http://hdl.handle.net/11349/6916 